\title{
La sostenibilidad del Sistema Nacional de Salud en España
}

\author{
The sustainability of the Spanish National Health System
}

JoséJ esús M artín M artín ${ }^{1}$

Maria del Puerto López del Amo González ${ }^{1}$

${ }^{1}$ Departamento Economía Aplicada, Facultad de CienciasEconómicasy Empresariales. Campus Universitario de Cartuja s/n 18011 Granada Spain.

jmartin@ugr.es
Abstract The Spanish National Health System (SNHS) has sustainability problems resulting from weaknesses in institutional design and governance compounded by the economic crisis it faces. The global economic crisis has had a particularly virulent impact in Spain, characterized by high levels of unemployment and public and private debt. Fiscal adjustment policies implemented may significantly compromise the SN HS. Along with general funding problems, the strong territorial decentralization of health jurisdictions in the Autonomous Communities has not been backed up by efficient Statelevel health coordination. The SN H S suffers from problems in its rules of governance, its autonomous financing system, human resource policies and diversity of direct and indirect management models in different Autonomous Communities. A reform strategy in Spanish healthcare governancemust be articulated within the context of a broader review of public policies to stabilize the lines of defense of the welfare state. Within the scope of the health sector, the financing system must be improved and institutional changes to increase efficiency must be implemented.

Key words Sustainability, Spain, H ealthcare system, Economic crisis, Decentralization
Resumen El Sistema Nacional de Salud (SN S) español presenta problemas de sostenibilidad derivados de deficiencias en su diseño institucional y de gobierno, agravadas por la crisis económica que padece. La crisis económica mundial ha tenido una repercusión especialmente virulenta en España, caracterizada por altos niveles de desempleo y dedeuda pública y privada. Las políticas de ajustefiscal emprendidas pueden deteriorar el SN S significativamente. Junto a problemas de financiación general, la fuerte descentralización territorial de competencias sanitarias en las Comunidades Autónomas no ha sido acompañada de un marco eficaz de coordinación sanitaria a nivel de Estado. EI SN S adolece de problemas en sus reglas de gobierno, su sistema de financiación autonómica, las políticas de recursos humanos y la diversidad de formas de gestión directa e indirecta que funcionan en las distintas Comunidades Autónomas. U na estrategia de reformas en el gobierno de la sanidad española debe articularse en el marco de una revisión más amplia de las políticas públicas que permita estabilizar las líneas de defensa del Estado del Bienestar. En el ámbito del sector sanitario se debe mejorar su sistema de financiación y desarrollar cambios institucionales para aumentar la eficiencia.

Palabras clave Sostenibilidad, España, Sistema Sanitario, Crisis económica, Descentralización 


\section{Introducción}

A principios del siglo XX, la muerte era un suceso cotidiano en España, la esperanza de vida de un recién nacido erainferior a la desus padres. Alterar este hecho natural y lograr que los padres mueran antes que los hijos ha sido una conquistatan revolucionaría como reciente. En el último siglo prácticamente se ha duplicado la esperanza de vida, ganando cerca de cuarenta años de vida por persona, lo que ha permitido recuperar gran parte del atraso histórico respecto a los países europeos. Pasar de tener una esperanza de vida de 40 años en 1910 a prácticamenteel dobleen el momento actual, modifica de forma radical la planificación de la vida y la estructura dela sociedad. Esta revolución demográfica es tan drástica como posiblementeirrepetible.

Naturalmente, no es posible atribuir esta mejora de la longevidad al sistema sanitario en exclusiva, han sido el crecimiento económico y las mejoras en la nutrición, los factores fundamentales de esta histórica caída de la mortalidad en todos los países desarrollados ${ }^{1}$. Un círculo virtuoso, dado que por ejemplo sin la disminución drástica de la tasa de mortalidad infantil, hubiera sido impensable la participación de la mujer en el mercado laboral.

Esto no quiere decir que el sistema sanitario de un país no importe, al contrario la evidencia internacional sugiere que el crecimiento económico no mejora lasalud sin una intervención pública explícita². El tipo de sistema sanitario no es neutral, sino un elemento clave en la calidad de vida delas personas, contribuyendo decisivamentea proteger a los más débiles de la sociedad dela incertidumbre y la angustia, de la enfermedad y el dolor. No cualquier actuación es válida, es una falacia sostener que cualquier incremento del gasto sanitario se traduce en mejoras de salud de la población. Los diseños institucionales -el buen gobierno sanitario- tienen un papel clave en evitar queel incremento del gasto sanitario sedisipe sin repercusiones positivas en la salud o la calidad de vida ${ }^{3}$.

El Sistema Nacional de Salud (SN S) español con una cobertura prácticamente universal, financiado por impuestos y con un alto nivel de servicios prestados, es una de las vigas maestras que, junto a las pensiones y el resto de políticas sociales, configuran el modesto Estado del Bienestar construido en las últimas décadas. Una red de seguridad para afrontar las incertidumbres de la vida tan necesaria como frágil, que está siendo cuestionada por la crisis económica y par- ticularmente por la estrategia de salida impuesta por los mercados financieros. Por primera vez en varias generaciones, los españoles contemplan la posibilidad de un futuro peor que su presente.

El artículo discutela sostenibilidad del SNS y sugiere algunas estrategias de reformas. El primer epígrafe caracteriza la crisis económica en España, contextualizando los problemas de sostenibilidad del SN S. Los dos siguientes epígrafes caracterizan algunos parámetros básicos del sistema sanitario, el gasto, la salud y el marco institucional y organizativo. Finalmente, el último epígrafe plantea al gunas líneas estratégicas dereforma del SN S para garantizar su sostenibilidad.

\section{Lacrisis económica}

Las crisis financieras son consustanciales al sistema económico capitalista, sólo entre 1970 y el año 2008 se han producido en el mundo 447 crisis financieras nacionales ${ }^{4}$. La actual crisis económica, por su profundidad y naturaleza global guarda indudables parecidos con la Gran Depresión de 1929, que fue superada tras una guerra mundial y la consagración de las ideas keynesianas, que otorgaban un papel protagonista al Estado y sometía a unas estrictas políticas de regulación del sector financiero ${ }^{5}$.

Durante treinta años reinó una indiscutible hegemonía de las políticas económicas keynesianas que finalizó con la crisis económica de los años setenta, iniciándose un cambio de rumbo en el pensamiento económico y político, con el ascenso de las ideologías neoliberales que representaban Ronald Reagan y Margaret Thatcher. El resurgir de la nueva economía neoclásica volvió a postular la eficiencia de los mercados, particularmentelos financieros, la hiperracionalidad de los agentes económicos, y la necesidad de que el Estado se retirara de la economía, para permitir el funcionamiento eficiente dela mano invisible del mercado.

Durante las últimas décadas la teoría económica estándar ha desarrollado sofisticados modelos matemáticos para demostrar la bondad de los mercados, en particular la hipótesis de los mercadosfinancieros eficientes, en comunión con la hipótesis de las expectativas racionales. Este marco teórico tan pletórico de sofisticación matemática como ayuno de sentido de la realidad, ha ignorado los sólidos resultados que no cuadraban con su esquema axiomático y ha servido de cobertura para desmantelar las regulaciones y controles financieros producto de las enseñan- 
zas de La Gran Depresión 6 . Enseñándose como verdad indiscutible en todas las facultades y escuelas de negocios del mundo, facilitó la ceguera colectiva sobre la catástrofe que se avecinaba. Casi nadie en el mundo académico, empresarial o financiero vio venir al lobo. El fracaso intelectual dela ciencia económica están dar hasido épico, contribuyendo a aumentar la percepción de quela economía es una ciencia forense, queraramente diagnostica la enfermedad cuando el paciente está vivo, sólo post mortem ${ }^{7}$.

En España, la integración en la zona euro posibilitó un largo período de tipos de interés bajos, lo queunido al diferencial deinflación con los países centrales del euro, alimentó una orgía consumista financiada con endeudamiento, que ha llegado a alcanzar el $114 \%$ del PIB. El ciclo expansivo basado en actividades de bajo valor añadido, como la construcción de viviendas, se sostuvo en la extraña creencia en unos mercados inmobiliarios y bursátiles eternamente al alza. España, al igual que otros países, construyó una inmensa burbuja, repleta de codicia, y amor por el dinero fácil, que al estallar puede llevarse por delante el sueño de una sociedad de bienestar, sustituyéndolo por una sombría pesadilla.

M ientras tanto la sociedad española se ha acostumbrado a convivir con el fraude fiscal, la economía sumergida (entre el 20 y el $25 \%$ del PIB) y el dinero negro como forma de pago habitual en distintas actividades ${ }^{8}$. Adicionalmente, durante las últimas décadas, tanto con gobiernos conservadores como de izquierdas, se ha desarrollado una política fiscal que difícilmente puedesostenerse desdeel principio detributar en proporción a los ingresos obtenidos. Un sistema fiscal claramente sesgado a favorecer a los que más tienen, que penaliza a las rentas salariales frente a las de capital, y no estimula la construcción de una moral fiscal en la ciudadanía, en el que el fraude fiscal sea contemplado como un delito y una miseria ética.

Con un déficit público que alcanzó el 11,4\% en 2009 , una tasa deparo del $20 \%$, un al to nivel de fraudefiscal denatural eza estructural y unosmercados financieros que dudan dela capacidad dela economía española desalir dela ratonera, el futuro no va a resultar sencillo. Sin política monetaria propia que permita una estrategia de salida basada en la devaluación, laúnica opción queconsideran los mercados es un ajuste severo de las políticas de gasto público, y una liberalización de los mercados, particularmente el laboral. Estas políticas sin embargo pueden conducir a la economía española a un estancamiento económico de larga duración. No deja de ser paradójico que los responsables de la crisis estén trasladando los costes de la misma a los grupos sociales más desfavore cidos, mientras siguen actuando en un mercado globalizado ante la impotencia de los gobiernos. El caso español es de manual, un gobierno socialista está aplicando duras políticas de ajuste para calmar a los mercados, enajenándose en el interregno su base electoral, y sin garantías de quelos mercados acepten el sacrificio.

En esta vorágineel SNS, uno de los principales logros de la sociedad española en las últimas décadas, afronta la crisis económica lastrado por significativos problemas financieros y de gobierno.

\section{Salud y gasto sanitario}

EI SN S español obtiene excelentes resultados con un gasto agregado relativamente modesto. La esperanza de vida de los españoles es de las más altas de Europa, 76,96 años para los hombres y 83,84 años para las mujeres. La esperanza de vida a los 65 años al canza como promedio casi 21 años entre las mujeres y 16 años en los varones, hecho conocido como "envejecimiento del envejecimiento", un fenómeno eminentemente femenino ${ }^{9}$.

No obstante, persisten importantes desigualdadesen salud entregrupos sociales y regiones ${ }^{10-14}$, sin que en las últimas décadas se haya producido un proceso deconvergenciaen salud en el conjunto del Estado Españo| ${ }^{15,16}$. Los resultados clínicos son homologables a los de los países más avanzados ${ }^{17}$ con un coste de los más bajos de la Unión Europea-15 en relación al PIB y el segundo más bajo en términos de gasto per cápita ${ }^{18}$. Constituye asimismo uno de los principales sectores económicos del paísque sólo en el ámbito público emplea a más de 1 millón de personas y gestiona un presupuesto cercano a los 60.000 millones de euros ${ }^{19}$.

La fuerte descentralización del sistema sanitario otorga la principal responsabilidad del gasto a las Comunidades Autónomas (CCAA) suponiendo el $5,05 \%$ del PIB demedia en 2005, y un $34,26 \%$ del gasto público total en el conjunto de las CCAA. Durante los años de expansión económica el gasto sanitario público delas CCAA se incrementó a una tasa media anual cercana al $9 \%$, superior al crecimiento nominal del PIB. Entre 1999 y 2005, el gasto medio por persona protegida tuvo un crecimiento anual medio del 7,16\% llegando a 1.091€ per cápita ${ }^{20}$.

La crisis económica ha reducido notablemente la tasa decrecimiento del gasto ${ }^{21}$. Los presupuestos iníciales de las CCAA disminuyen $4,11 \%$ de 
promedio en el año 2011 en relación al 2010. El presupuesto per cápita nacional (media de las CCAA) es de 1.289 - con notables variaciones entre regiones con máximos en País Vasco ( 1.564 •) y mínimos en Baleares $\left(1.003 \cdot{ }^{222}\right.$.

El sistema sanitario público tieneuna tendencia secular a gastar por encima de los presupuestos autorizados por los parlamentos autonómicos, dando lugar periódicamente a operaciones extraordinarias de saneamiento del déficit acumulado por las CCAA por parte del gobierno centra| ${ }^{23}$. Dada la fuerte inercia del gasto sanitario a crecer por encima del crecimiento nominal del PIB, es muy probable que se esté creando un importante déficit no reconocido, cuyo importe global y desagregado para cada CA se desconoce. La opacidad del sistema de información sobre el gasto público de las CCAA es uno de los motivos de la actual desconfianza de los mercadosfinancierosen la capacidad deEspaña dehacer frente a su deuda pública, al suponer que hay importantes desajustes entre el gasto reconocido y el gasto real devengado.

El recientemente aprobado sistema de financiación autonómica ${ }^{24}$, a pesar de haber avanzado en al gunos de los problemas seculares que arrastra el desarrollo institucional dela descentralización territorial de España, sigue padeciendo de importantes problemas que posiblemente van a lastrar su desarrollo futuro ${ }^{25}$. Entre ellos pueden mencionarse, su carácter complejo y opaco; su dependencia dela recaudación detributos regionales, muy ligados al ciclo económico, lo quepue de producir asfixia financiera en épocas de recesión o débil crecimiento económico; la inexistencia decriterios sólidos de definición de necesidad relativa en sanidad; la inexistencia asimismo de un carácter finalista de los recursos sanitarios, lo quedificulta queel Estado pueda garantizar unos niveles de gasto sanitario público per cápita no excesivamentedispersos entreterritorios; y finalmente, aunque no menos importante, la insuficiencia de los mecanismos establecidos para la corrección de las desigual dades sociales en salud.

Varios estudios han llevado a cabo proyecciones del gasto sanitario público para España en las próximas décadas ${ }^{26-32}$, y aunque sus resultados difieren en función de la metodología empleada, hay un consenso sobre algunas cuestiones.

En primer lugar, el crecimiento del gasto sanitario público derivado del impacto demográfico (aumento de la población y envejecimiento) será con una alta probabilidad, inferior al crecimiento del PIB, y en todo caso sostenible a unas tasas moderadas de crecimiento, y en segundo lugar, el aumento dela proporción del PIB destinado a la financiación sanitaria depende del ritmo de aumento de factores endógenos, más dependientes de la gestión, como los precios relativos de los inputs, el cambio tecnológico, las nuevas prestaciones, la mayor utilización de servicios o el aumento de la intensidad de recursos por acto médico.

$\mathrm{N}$ inguno de estos trabajos considera en sus proyecciones la profunda crisis económica actual y las políticas de consolidación fiscal que se están produciendo, que implican un crecimiento nulo 0 incluso negativo del gasto sanitario público en los próximos años. Esta situación va a someter al SN S a considerablestensiones y al riesgo de un deterioro claro de la oferta de servicios sanitarios públicose incluso a un proceso deprivatización acelerada de los mismos, como ya está sucediendo en ciertas CCAA. Es por tanto previsible que las severas restricciones presupuestarias de los próximos años supongan un deterioro, tanto de la inversión en tecnologías, como de las rentas de los profesionales sanitarios, así como de la instrumentalización de políticas orientadas a corregir las desigualdades sociales en salud.

\section{El Sistema Nacional de Salud}

El actual sistema sanitario público español surge históricamente a mediados del siglo pasado inspirado en los modelos continentales europeos de Seguridad Social, aunque a diferencia de ellos crea una extensa red propia de centros y organizaciones propias de carácter ambulatorio y hospitalario. El INSALUD (Instituto $\mathrm{Na}$ cional dela Salud), creado en 1978 como un órgano gestor de la ASSS, acogió esta estructura de oferta, configurándose como una gran organización fuertementecentralizada que daba cobertura sanitaria directa a más del $84 \%$ dela población española.

Realizada la transición democrática, y aprobada la Constitución de 1978, la Ley General de Sanidad de 1986 inició el proceso detransformación del sistema deSeguridad Social en un modelo tipo Sistema N acional deSalud, definido en su artículo 44.2 como el conjunto de los Servicios de Salud de la Administración del Estado y delos Servicios de Salud de las CCAA en los términos establecidos en la presente Ley identificando como principios fundacionales, la universalidad en el acceso, la descentralización en la gestión, la equidad en el acceso a los servicios y prestaciones, la financiación pública y la participación social. 
Deforma progresiva el sistema sanitario evolucionó desde un modelo peculiar de Seguridad Social a un modelo financiado por impuestos generales, de cobertura casi universal, que coexiste con un importante sector de seguros privados que dan cobertura a uno de cada cinco españoles. Esto, por un lado permite una disminución de la presión de demanda de servicios sanitarios en el ámbito público, pero por otro posibilita estrategias detraslado de costes del sector asegurador privado al sector público.

La Ley General deSanidad otorgó a las CCAA la responsabilidad exclusiva de la organización de sus servicios de salud, creando progresivamenteuna estructura fuertemente descentralizada de organización y gestión sanitaria que se inició en 1983 con la creación del Servicio Vasco de Salud/O sakidetza y concluyó en el año 2001 con la constitución del Servicio Cántabro de Salud.

Esta estructura descentralizada no ha ido acompañada de una arquitectura institucional acordecon las necesidades degobernación del SNS, de forma que en la práctica, éste SNS no va más allá de la suma de las actuaciones de los distintos Servicios Regionales de Salud y de los acuerdos adoptados por unanimidad en el Consejo Interterritorial del SNS. El déficit de gobernanza del Estado en el SN S se ha manifestado en importantes déficits, tanto de eficiencia como de equidad ${ }^{33}$.

Esta "anorexia" institucional del Estado, ha tratado de resolverse con la Ley 16/2003 deCohesión y Calidad del SN S, queen su artículo 2 recoge tanto el objetivo de igualdad efectiva y de calidad en la prestación de los servicios en todo el territorio del Estado, como el principio decoordinación y la cooperación de las administraciones públicas para la superación de las desigualdades en salud. La Ley 16/2003 define seis ámbitos de colaboración entre el Estado y las CCAA: prestaciones del sistema, farmacia, profesionales, investigación, sistema de información y calidad.

Su desarrollo ha permitido algunos avances en la coordinación del sistema, en particular la aprobación en 2006 del primer Plan de Calidad del SNS 2006-2010, o los recientes acuerdos del Consejo Interterritorial del SN S del 18 de marzo de 2010, aprobados en un entorno de crisis económica e insuficiencia presupuestaria, que plantean 24 medidas para garantizar la coordinación y sostenibilidad del sistema. La mayoría de estas medidas no obstante están formuladas de forma tan genérica que difícilmente pueden estructurar un plan ambicioso de reformas ${ }^{34}$.

Uno de los principales problemas de coordinación se da en la política de recursos humanos, manifestándose en las políticas retributivas, el desarrollo de la carrera profesional, los desajustes en la oferta y demanda de profesionales médicos y deenfermería y la movilidad de los mismos.

España cuenta actual mente con 325 médicos en activo por cada 100.000 habitantes, es decir un total de 144.379 profesionales. De ellos, 141.410 serían especialistas, trabajando la mayoría (104.598) para el SN S19. A pesar de este ratio, superior a los estándares internacionales, existe un déficit en algunas especialidades médicas como medicina familiar, pediatría, anestesiología y reanimación, o cirugía ortopédica y traumatología. Este déficit está motivado por una organización asistencial con patrones rígidos de funcionamiento, y un número relativamente exiguo de horas anuales trabajadas.

El ámbito de las relaciones laborales está regulado por un marco de naturaleza funcionarial específico continuista con el marco de regulación laboral preexistente históricamente, aunque delegando una parte considerable de las competencias en cada una de las 17 CCAA (Ley 55/2003) ) $^{35}$

Laculminación en 2002 del proceso detransferencias de la Asistencia Sanitaria de la Seguridad Social (INSALUD) a las CCAA generó una dinámica inflacionista en las rentas salariales del sector. Las negociaciones entre los políticos autonómicos y los representantes sindicales dieron lugar a un proceso continuo de reivindicaciones en el que en cada Comunidad Autónoma se tomaba como re ferenteel mejor acuerdo existente en las demás.

Las actuales exigencias de los mercados financieros deuna política de consolidación fiscal, como condición para seguir financiando la deuda pública y privada española a tipos de interés sostenibles, no sólo ha acabado con las alegrías retributivas de los últimos años, sino que dibuja un horizonte de pérdida de rentas de los profesionales del sector sanitario público, unido a una previsible destrucción de puestos de trabajo. Duranteel ejercicio 2010, seaplicó una reducción salarial del $5 \%$ de media, al igual que al resto de trabajadores de las Administraciones Públicas. Para este año 2011 se han congelado las retribuciones al conjunto de trabajadores públicos.

Otro rasgo característico del SNS es la fuerte innovación organizativa acaecida en las últimas décadas que se ha sustantivado en cuatro estrategias:

(1) El desarrollo de estrategias gerencialitas mediante la introducción de contratos programa y contratos de gestión, en los servicios regionales de salud, unido al desarrollo de la gestión clínica y de la gestión de procesos. 
(2) La trasformación en empresas públicas de los Servicios de Salud de varias CCAA frentea su caracterización habitual de organizaciones sujetas a derecho público, aunque con personalidad jurídica propia.

(3) La dotación de personalidad jurídica individual a hospitales y centros sanitarios nuevos mediante distintas fórmulas, fundamentalmente empresas públicas, fundaciones públicas y consorcios.

(4) El desarrollo de formas de contratación externa o gestión indirecta dela atención sanitaria pública con proveedores privados comerciales, mediantefórmulas de "concesión administrativa" de atención a toda la población de un Área de Salud. Estas fórmulas concesional es se están desarrollando únicamente en dos CCAA, Valencia y Madrid, gobernadas desde hace años por el ala más liberal del Partido Popular. Es un modelo de partenariado público privado, (PPP, Public Private Partnership) que va un paso más allá de la PFI (Private Finance Initiative), al concesionar externamente también los servicios médicos, y no únicamente la construcción, instalaciones, mantenimiento y servicios generales, que es lo que incluía el sistema original británico de PFI. Estas concesiones administrativas, conocidas en España como "modelo Alzira" por la localidad valenciana donde se inició este sistema, es una fórmula de gestión indirecta por el que la empresa concesionaria recibe de la Comunidad Autónoma un pago (canon) durante todo el período de vigencia de la concesión, generalmente 30 años. Estecanon está formalizado como un pago capitativo por cada habitante del ámbito territorial cubierto por los servicios de salud concesionados. Su opacidad y falta de transparencia respecto a la cuantía y a su desglose han sido objeto de duras críticas, reforzadas por el hecho de que estos modelos nunca hayan sido evaluados y de disponer de información muy escasa e incompleta sobre los mismos.

\section{Reformas}

La actual crisis económica, ha espoleado el debate preexistente sobre la sostenibilidad del SN ${ }^{36}$, generando un importante número de trabajos que si bien no coinciden ni en diagnostico ni en soluciones, si parecen compartir cierto horizonte apocalíptico, síntoma de la profunda crisis que atraviesa el país, una de las más importantes de su historia reciente $\mathrm{e}^{17,33,34,37-39}$.

Dado su carácter sectorial, estos estudios no plantean la interrelación entre la sostenibilidad del SN S y el resto de políticas sociales. Las estrategias de reformas sanitarías deben enmarcarse en una estrategia más amplia de reforma institucional del Estado, sin la que su sostenibilidad estaría comprometida. La situación exige un amplio aliento regeneracionista, que involucre a todas las fuerzas políticas y sociales en un Pacto de Estado $^{40}$, que permita soportar los considerables sacrificios y pérdida de rentas que esperan a la mayoría de la población. Un pacto que no haga recaer los ajustes, sobre la clientela habitual, es decir los más desfavorecidos y débiles. Como elementos claves de esta estrategia de reforma deben señalarse: una urgente reforma fiscal orientada a regenerar la moral fiscal de la sociedad, en la que la evasión y la ilusión fiscal no sea el pan nuestro de cada día; una reforma de la financiación de los partido políticos, para acabar con un sistema opaco, placenta de todo tipo de corrupciones; una reforma de la arquitectura institucional y territorial del Estado presidida más por la racionalidad y el principio deigualdad quepor los sentimientos de pertenen cia o las necesidades de mayorías parlamentarías, y una ambiciosa reforma de las Administraciones Públicas a todos los niveles territoriales, que profesionalicela gestión pública y destierrelas tentaciones clientelares de los partidos políticos?.

En el ámbito específico del sector sanitario existen dos estrategias básicas para garantizar la sostenibilidad del SN S: aumentar los ingresos fiscales dedicados a esa finalidad, y aumentar la eficiencia del sistema de provisión sanitaria.

En relación a la primera, frentea la opción de crear sistemas de copago sanitario como defienden algunos estudios, es preferible, tanto en términos de eficiencia como dejusticia, la opción de impuestos finalistas, en el marco de la fundamental reforma de la fiscalidad mencionada anteriormente. Por ejemplo, la creación de un Fondo de I gualdad del SNS de carácter estatal, cuyos recursos se utilizarían para financiar políticas de salud orientadas fundamentalmente a la corrección de desigualdades y a la lucha contra los principales causantes de la morbi-mortalidad en España: enfermedades cardiovasculares, cáncer, diabetes, etc. Se financiaría con recursos fiscales de carácter final ista y sería gestionado por una agencia con personalidad jurídica propia y autonomía en la que estarían representados las CCAA y el Gobierno Central. Esta opción permitiría al Estado intervenir directamente en toda España en función de planes previamente definidos, y recuperar cierto poder de gasto y orientación de políticas como sucede en otros países ${ }^{41}$. 
En relación a la mejora dela eficiencia del SNS pueden apuntarse algunos elementos clave para mejorar la gobernanza del sistema. En primer lugar, un Código deBuen Gobierno del SNS, que más allá de la retórica, establezca los principios para la gobernanza, transparencia y la rendición decuentas.

En segundo lugar, el desarrollo de un sistema de información integrado del SNS. La información sanitaria es un bien público puro, que debe producirse al nivel y calidad adecuada, evitando tanto el parasitismo oportunista (free-rider) como el riesgo moral (moral hazard) a nivel organizativo e institucional. La creación de una Agencia de Información Estatal con personalidad jurídica propia y participación de las CCAA y el M inisterio de Sanidad y Consumo podría ser la solución.

En tercer lugar, se necesita una política estratégica integrada de salud pública a nivel estatal, que impida rupturas unilaterales de una CA sobre cuestiones que generen externalidades negativas sobre las poblaciones de otras CCAA, como por ejemplo la política de vacunaciones. Esta política debe así mismo desarrollar estrategias para minimizar los efectos negativos del cambio climático en la salud ${ }^{42}$.

En cuarto lugar, para impedir que las desigualdades de oferta se traduzcan en desi gualdades de acceso y uso, el mejor camino es protocolizar la atención sanitaria mediante guías clínicas a nivel nacional, y la creación de una Agencia Nacional deCalidad Clínica y Evaluación de Tecnologías, inspirada en el $\mathrm{N}$ ational Institute for Clinical Excellence(NICE) inglésy en el Instituto para la Calidad y Eficiencia Económica en la Sanidad (IQWiG) de Alemania.

En quinto lugar, deben desarrollarse marcos institucional esy organizativosqueintegren servicios sociales y sanitarios. El envejecimiento poblacional y el predominio de las enfermedades crónicas dificultan tanto la medida de la salud como la respuesta de los servicios sanitarios, cuya oferta seha estructurado históricamentepara responder a patologías agudas. Se debe diseñar un plan estratégico que reorientelos servicios sanitarios para dar respuesta a problemas de enfermedad que permanecen en el tiempo y en el que la atención hospitalaria sólo puede jugar un papel limitado, al menos en su forma convencional.

En sexto lugar, es imprescindible un acuerdo sobre política de recursos humanos a nivel estatal que junto a un pacto de rentas, proponga un nuevo escenario para la formación, selección y retribución de médicos y otros profesionales de la sanidad, en el queel profesionalismo y la vocación sustituyan al excesivo énfasisquehasta ahora se ha puesto en las recompensas exclusivamente monetarias. Este pacto debe incluir una profesionalización urgente de la gestión sanitaria.

Finalmente, en relación con las innovaciones organizativas y de gestión de las últimas décadas, el frenesí reformador debe dejar paso a la evaluación. Es necesario realizar estudios sistemáticose independientes quepermitan una evaluación comparativa de los resultados que obtienen las nuevas formas de gestión directa frente a las formas burocráticas degestión pública predominantes en el sector. La personificación jurídica de centros sanitarios públicos debe ir acompañada de cambios en el marco de regulación y en el entorno organizativo que hagan que sus potenciales ventajas se consoliden, mientras se minimizan los potenciales efectos negativos que pudieran tener en términos de equidad e igualdad de acceso.

En relación con las nuevas formas de gestión indirecta se ha de ser particularmente cauteloso, dadas las implicaciones negativas que un crecimiento desmesurado de las mismas pudiera tener. La propiedad privada lucrativa se caracteriza por la apropiación privada del excedentecomo remuneración residual del propietario. Cumplen una función socialmente útil cuando premian la eficiencia, el riesgo dela utilización del capital o la innovación. Estas funciones del beneficio solamente se dan cuando no hay fracasos graves de mercado, como sucede en sanidad.

Estetipo de experiencias degestión indirecta, si bien pueden constituir un factor de innovación, deben estar sujetas a unos mecanismos de regulación y control deuna extraordinaria transparencia eindependencia que dificulten las posibilidades de colusión de intereses. En cualquier caso deben tener un carácter minoritario en relación con otros proveedores públicos o privados no lucrativos, de forma que los intereses por la rentabilidad y el beneficio no predominen en la definición de las políticas públicas ${ }^{43}$.

España está atravesando una de las mayores crisis de la historia reciente y existe el riesgo no sólo de no avanzar en salud, sino incluso de retroceder, si la crisis económica sealarga en el tiempo. Las crisis económicas profundas y de larga duración tienen como resultado el empeoramiento de los indicadores de salud, en particular en los colectivos socialmente más desfavorecidos ${ }^{44}$. Podemos asistir a un creciente desajuste entre necesidades sanitarias y posibilidades de crecimiento del gasto sanitario público, al menos a corto plazo. 
Para mitigar esta situación se debe establecer un plan ambicioso de reforma institucional, que orienteel sistema sanitario en el sentido anteriormente enunciado. Puede ser no obstante insuficientesi no seenmarca en un proceso más amplio de reformas de la políticas públicas a nivel del conjunto del Estado 7 . Como recoge Jon Elster en su excelente libro U vas amargas ${ }^{45}$, citando una oración atribuida a los alcohólicos anónimos: Ojalá tengamos serenidad para aceptar lo que no podemos cambiar, coraje para cambiar lo que podemos y sabiduría para reconocer la diferencia.

\section{Colaboradores}

Martín JJ y López del Amo MP han trabajado conjuntamente en la concepción, redacción y re visión.

\section{Referencias}

1. Fogel RW. The escape from unger and premature death, 1700-2100. Cambridge: Cambridge University Press; 2004.

2. Cutler $D$, Deaton A, Lleras-Muney A. The determinants of mortality.J Economic Perspectives 2006; 20(3): 97-120. (NBER Working Papers, n. 11963).

3. Ortún V, director. El buen gobierno sanitario. Madrid: Springer Healthcare Communications; 2009.

4. Laeven L, Valencia F. Systemic banking crises: a new database. Washington: Fondo Monetario Internacional; 2008. (Contract WP08/224).

5. Skidelsky R. Keynes: the return of the master. Essex: Allen Lane Penguin Books; 2009.

6. Stiglitz J. Freefall: America, free markets, and the sinking of the world economy. New York: WW Norton \& Company; 2010.

7. Martín JJ. Crisis económica y sostenibilidad del Sistema Nacional de Salud. El M édico 2010; 1109:16-19.

8. Tax Justice Network. Fraude fiscal en España. Red de Justicia Fiscal [periódico na Internet]. 2008 Set. [acceso en 12 enero 2011]. Disponible en: http:// www.redjusticiafiscal.org/competencia-fiscal/elfraude-fiscal-en-espana.html

9. España. M inisterio de Sanidad y Consumo. La salud de la población española en el contexto europeo del Sistema $\mathrm{N}$ acional de Salud: indicadores de salud. M adrid: Ministerio de Sanidad y Consumo; 2005. [acceso en 12 enero 2011]. Disponible en: http://www.msc.es/ estadEstudios/estadisticas/inforRecopilaciones/tabla/ Indicadores5.pdf

10. Cantarero D, Pascual M. Impacto de la desigualdad de la renta sobre los indicadores de salud: un estudio empírico. In: Actas del VII Encuentro de Economía Aplicada; 2004; Vigo, España [acceso en 9 enero 2010]. Disponible en: http://www.revecap.com/ encuentros/anteriores/viieea/autores/C/29.doc 
11. González B, Barber P. Desigualdades territoriales en el Sistema Nacional de Salud (SNS) de España. Documento de trabajo Fundación Alternativas 2006; 90.

12. Regidor $E$, Martínez D, Astasio P, Ortega P, Calle ME, Domínguez V. Evolución de las desigualdades socioeconómicas y las desigualdades en la percepción de la salud en España. Gaceta Sanitaria 2006; 20(3):1-5.

13. Montero R, Martín JJ, Jimenez JD. Inequidad horizontal en el acceso a servicios sanitarios y nive educativo en España. En: Cabasés JM, coordinador. La financiación del gasto sanitario en España: valoración del sistema de financiación, medida de la necesidad relativa y equidad. Bilbao: Fundación Banco Bilbao Vizcaya Argentaria; 2010.

14. Martín JJ, Amo Gonzáles M PL, M elchior JM. Características individuales, capital social y privación en el estado de salud percibido en España. En: Cabasés JM, coordinador. La financiación del gasto sanitario en España: valoración del sistema de financiación, medida de la necesidad relativa y equidad. Bilbao: Fundación Banco Bilbao Vizcaya Argentaria; 2010.

15. Montero R, Jimenez JD, M artín JJ. Decentralisation and convergence in health among the provinces of Spain (1980-2001). Soc Sci M ed 2007; 64(6):12531264.

16. Regidor E, Martínez D, Astasio P, Ortega P, Calle ME, Domínguez V. Evolución de las desigualdades socioeconómicas y las desigualdades en la percepción de la salud en España. Gaceta Sanitaria 2006; 20(3):1-5

17. Beltrán A, Forn R, Garicano L, Martlnez M, Vázquez $\mathrm{P}$. Impulsar un cambio posible en el Sistema Sanitario. Madrid: M cKinsey \& Company y FEDEA; 2009 [documento na Internet]. [acceso en 9 enero 2011]. Disponible en: http://www.cambioposible.es/ documentos/sanidad cambio posible.pdf

18. OECD Health Data 2010. Selected data. Total expenditure en health \% of Gross Domestic Product - Total health expenditure per capita US\$ PPP [documento na Internet]. [acceso en 11 enero 2011]. Disponible en: http://stats.oecd.org/Index.aspx?D ataSetC ode $=$ HEALTH

19. España. M inisterio de Sanidad y Consumo. Recursos del SN S: presupuestos iniciales para sanidad de las comunidades autónomas, la administración central y la seguridad social (2007-2010). [documento na Internet]. [acceso en 9 enero 2010]. Disponible en: http://www.msc.es/estadEstudios/estadisticas/docs/ RecursosRed2010.xls

20. Instituto de Estudios Fiscales. Informe para el análisis del gasto sanitario (IAGS). Madrid: Secretaría de Estado de Hacienda y Presupuestos; 2007. (Colección Libros Blancos).

21. Espanha. M inisterio de Sanidad, Política Social e I gualdad. Recursos del Sistema Nacional de Salud: presupuestos iniciales para sanidad de las comunidades autónomas, la administración central y la seguridad social 20072010 [acceso en 12 enero 2011]. Disponible en: http:/ /www.msps.es/gl/estadEstudios/estadisticas/docs/ RecursosRed2010.xls
22. Federación de Asociaciones para la Defensa de la Sanidad Pública. Los presupuestos sanitarios de las CCAA para 2011. [documento na Internet]. [acceso en 9 enero 2011]. Disponible en: http://www.nodo50. org/fadsp/pdf/preCCAA11.doc

23. M artín JJ. Balance crítico de la descentralización territorial de la sanidad. Colección M editerráneo Económico [periódico na Internet]. 2006. [acceso en 12 enero 2011]; 10: 385-404. Disponible en: http://www. fundacioncajamar.es/mediterraneo/revista/me1018. pdf

24. Consejo de Política Fiscal y Financiera. Acuerdo 6/ 2009 de 15 de julio para la reforma del sistema de financiación de las comunidades autónomas de régimen común y ciudades con estatuto de autonomía [documento na Internet]. [acceso en 9 enero 2011]. Disponible en: http://www.meh.es/Documentacion/Publico/ PortalVarios/FinanciacionTerritorial/Autonomica/ AcuerdosConsejo/Acuerdo\%206 2009\%20Reforma \%20Sistema\%20Financiaci\%C3\%B3n.pdf

25. Cabasés JM, coordinador. La financiación del gasto sanitario en España: valoración del sistema de financiación, medida de la necesidad relativa y equidad. Bilbao: Fundación Banco Bilbao Vizcaya Argentaria; 2010.

26. Puig-Junoy J, Castellanos A, Planas I. Análisis de los factores que inciden en la dinámica del crecimiento del gasto sanitario público. Registro histórico y proyecciones 2004-2013. En: Puig-Junoy J, López-Casasnovas G, Ortún V. ¿M ás recursos para la salud? Barcelona: Masson; 2004.

27. Puig-Junoy J, Puig R. y Casado D, coordinadores. El impacto de la demografía sobre el gasto sanitario futuro de las comunidades autónomas. M adrid: Pfizer; 2009.

28. Ahn N, García JR, H erce JA. Demographic uncertainty and heath care expenditure in Spain: documento de trabajo 2005-07 [documento na Internet]. [acceso en 12 enero 2011]. Disponible en: http://www. fedea.es/pub/Papers/2005/dt2005-07.pdf

29. Hagist C, Kotlikoff LJ. Who's going broke? Comparing healthcarecosts in ten OECD countries. NEBER Working Paper 2005; 11833.

30. Oliveira Martins J, De la M aisonneuve C. Projecting OECD health and long term care expenditures: what are the main drivers? OECD Economics Department Working Paper [periodico na Internet] 2005 [acceso en 12 enero 2011]; 477. Disponible en: http:/ /www.oecd.org/dataoecd/57/7/36085940.pdf

31. European Commission. Economic policy and the European Commission: the impact of ageing on public expenditure - projections for the UE25 member states on pensions, health care, long-term care, education and unemployment transfers (2004-2050). [S.I.: s.n.]; 2006. Special report.

32. Sánchez Fernández J, Sánchez Maldonado J. Necesidades de gasto sanitario público inducidas por factores demográficos: un análisis por comunidades autónomas. Revista de Estudios Regionales 2009; 7:157-180. 
33. Repullo JR, Freire JM. Gobernabilidad del Sistema Nacional de Salud: mejorando el balance entre los beneficios y los costes de la descentralización. Informe SESPAS 2008. Gaceta Sanitaria 2008; 22(Supl.1):118-125.

34. Consejo Económico y Social (CES). Desarrollo autonómico,competitividad y cohesión social en el sistema sanitario. España:Imprenta Fareso; 2010. [Colección Informes, 1].

35. España. Ley 55 de 16 de diciembre de 2003 del Estatuto M arco del Personal Sanitario de los Servicios de Salud. Boletín Oficial del Estado [periódico en Internet] 2003; 301:44742-44763. [acceso en 2011 enero 9]. Disponible en: http://www.boe.es/boe/ dias/2003/12/17/pdfs/A44742-44763.pdf

36. Repullo JR, Oteo LA, editores. Un nuevo contrato social para un sistema nacional de salud sostenible. Barcelona: Ariel; 2005.

37. Del Llano J. Sanidades autonómicas isolución o problema? M adrid: Círculo de Sanidad; 2009.

38. Gil V, Barrubés J, Álvarez JC, Portella E. Sostenibilidad financiera del sistema sanitario: 10 medidas estructurales para afrontar las causas del crecimiento del gasto. Antares Consulting; 2010. [acceso en 9 enero 2011]. Disponible en: http://www.antares-consulting.com/ content/update/publicaciones/documentos/ 1306313081104045.pdf

39. Federación de Asociaciones para la Defensa de la Sanidad Pública. La sostenibilidad del sistema sanitario público: 12 propuestas para garantizarla. [documento na Internet]. [acceso en 9 enero 2011]. Disponible en: http://www.nodo50.org/fadsp/pdf/ 12propSostenibilidadSN S. doc

40. Repullo JR. ¿Entraría en un "Pacto de Estado" la revitalización de los cimientos legales de la sanidad española? El M édico 2009; 1103:12-15.

41. Martín JJ, Sánchez J. El fondo de igualdad del Sistema Nacional de Salud. En: Cabasés JM, coordinador. La financiación del gasto sanitario en España: valoración del sistema de financiación, medida de la necesidad relativa y equidad. Bilbao: Fundación Banco Bilbao Vizcaya Argentaria; 2010. p. 419-437.

42. Ballester F, Díaz J, Moreno JM. Cambio climático y salud pública: escenarios después de entrar en vigor el Protocolo de Kioto. Gaceta Sanitaria 2006; 20(Supl.1):160-174.

43. Martín JJ, López del Amo M P. Innovaciones organizativas y de gestión en el sistema nacional de salud: materiales formativos relativos al curso online sistemas sanitarios y nuevas formas de gestión, impartido por el Instituto de Postgrado de Estudios de M edicina. Madrid: Editrain Consultoría y Formación; 2010.

44. Dávila CD, González B. Crisis económica y salud. Gaceta Sanitaria 2009; 23(4):261-265.

45. Elster J. U vas amargas: sobre la subversión de la racionalidad. Barcelona: Edicions 62; 1988.

Artigo apresentado em 16/02/2011

Aprovado em 29/03/2011

Versão final apresentada em 14/04/2011 